\title{
Comparison of Acceptable Noise Levels Using Korean and Non-Semantic Speech Signals in Normal Hearing Subjects
}

\author{
Seon Geum Kim ${ }^{1,2}$, Kyung Jin Roh ${ }^{3}$, Ah Young Park ${ }^{1}$, Byung Il Choi ${ }^{1}$, \\ Seong Ah Hong, ${ }^{1}$ Jae Hee Lee ${ }^{2}$, and Eun Jin Son ${ }^{1}$ \\ ${ }^{1}$ Department of Otorhinolaryngology, Yonsei University College of Medicine, Seoul; and ${ }^{2}$ Department of Audiology, \\ Hallym University of Graduate Studies, Seoul; and ${ }^{3}$ Department of Otorhinolaryngology, Inje University College of Medicine, \\ Seoul Paik Hospital, Seoul, Korea
}

\begin{abstract}
정상 청력인에서 한국어 및 비의미 어음을 이용한 수용소음레벨 검사방법의 비교
김선금 ${ }^{1,2} \cdot$ 노경진 $^{3} \cdot$ 박아영 $^{1} \cdot$ 최병일 $^{1} \cdot$ 홍성아 $^{1} \cdot$ 이재희 ${ }^{2} \cdot$ 손은진 ${ }^{1}$

연세대학교 의과대학 이비인후과학교실, ${ }^{1}$ 한림국제대학원대학교 청각학과, ${ }^{2}$ 인제대학교 의과대학 서울백병원 이비인후과학교실 ${ }^{3}$
\end{abstract}

\author{
Received June 12, 2017 \\ Revised August 30, 2017 \\ Accepted September 5, 2017 \\ Address for correspondence \\ Eun Jin Son, MD, PhD \\ Department of Otorhinolaryngology, \\ Yonsei University \\ College of Medicine, \\ Gangnam Severance Hospital, \\ 211 Eonju-ro, Gangnam-gu, \\ Seoul 06273, Korea \\ Tel $+82-2-2019-3460$ \\ Fax $+82-2-3463-4750$ \\ E-mail ejson@yuhs.ac \\ Address for correspondence \\ Jae Hee Lee, PhD \\ Department of Audiology, \\ Hallym University of Graduate \\ Studies, 427 Yeoksam-ro, \\ Gangnam-gu, Seoul 06197, Korea \\ Tel +82-2-2051-2942 \\ Fax $+82-2-3453-6618$ \\ E-mail leejaehee@hallym.ac.kr
}

Background and Objectives The acceptable noise level (ANL) test measures the maximum noise level that a subject can tolerate while following speech. Given the limited investigation of ANL across different languages, this study aimed to compare ANLs using both Korean semantic speech and non-semantic speech signals (reversed Korean speech and international speech test signal, ISTS) in normal-hearing subjects.

Subjects and Method Twenty-five subjects with normal hearing (9 males, 16 females, with the mean age of $28.5 \pm 3.15$ years) were included in the study. ANLs were obtained using three speech materials (Korean speech, reversed Korean speech, and ISTS) as the target speech material and an eight-talker babble noise as the competing background noise.

Results Generally, the inter-subject variability was large. The ANLs did not differ statistically regardless of the type of speech signal.

Conclusion The ANLs seemed to be unaffected by the meaningfulness or semantic contents of the target speech, at least in normal-hearing adults. Thus, non-meaningful speech signals or non-semantic material can be considered as the target signal for the implementation of ANL test. Korean J Otorhinolaryngol-Head Neck Surg 2017;60(12):614-20

Key Words Acceptable noise level · Most comfortable level.

\section{Introduction}

The acceptable noise level (ANL) is a measure of the amount of background noise that subjects can tolerate when follow-

This is an Open Access article distributed under the terms of the Creative Common Attribution Non-Commercial License (http://creativecommons.org/licenses/by-nc/4.0) which permits unrestricted non-commercial use, distribution, and reproduction in any medium, provided the original work is properly cited. ing a speech signal. ${ }^{1)}$ For the ANL measurement, the maximum comfortable level (MCL) of speech signal is measured, and then the subject selects the maximum level of background noise level (BNL) that the subject is willing to accept. The ANL is calculated as the difference between MCL and BNL $(\mathrm{ANL}=\mathrm{MCL}-\mathrm{BNL})$. A lower ANL indicates that the subject tolerates more background noise, whereas a higher ANL 
means that the subject has a lower tolerance for noise.

Acceptance of noise and speech understanding in noise are thought to be distinct aspects of hearing in noisy environment. However, ANL measurement is generally accepted as a psychoacoustic test that examines the acceptance of noise while listening to and following discourse of the presented speech signal. The listener is not required to understand the speech content. In contrast, tests of speech perception in noise aim to assess the listener's ability to identify a target word or sentence in a noisy background. The ANL measures the listeners' intrinsic characteristics or intrinsic reactions to background noise in a controlled condition of listening to speech. ${ }^{2)}$ Accordingly, ANLs were shown to be unrelated to the age ${ }^{1)}$ or gender of listeners. ${ }^{1-4)}$ Contrary to the previous studies, which reported that the ANLs are unrelated to degree of hearing loss, ${ }^{1,2)}$ a recent report highlighted a significant, but weak, correlation between ANLs and hearing sensitivity in older adult listeners. ${ }^{5)}$

Extending from the previous studies that followed the "traditional' procedure using a male talker and babble background noise, as in the original ANL study of Nabelek, et al., ${ }^{1)}$ recent studies have evaluated different types of target speech and background noise. For example, the ANLs have been investigated using various languages such as English, ${ }^{1)}$ Korean, ${ }^{6}$ Mandarin, ${ }^{7)}$ Danish, and Swedish, ${ }^{8)}$ as well as non-semantic speech signals including reversed speech recordings or the international speech test signal (ISTS). ${ }^{8)}$ It remains unclear whether the language and intelligibility of the speech material affect ANLs.

Additionally, when controlling meaningfulness of the competing speech masker, the ANLs appeared to be dependent on the number of competing talkers for normal- hearing listeners. ${ }^{6)}$ Several studies have used non-semantic speech signals for ANL measurements, but the findings somewhat differed across studies. Brännström, et al. ${ }^{8)}$ and Olsen, et al. ${ }^{910)}$ used ANL materials including non-semantic signal such as ISTS and evaluated the listeners' performance. ${ }^{8-10)}$ They found a poor repeatability and significant differences between the Danish and Swedish populations, possibly depending on test sites and language. Ho, et al. ${ }^{7)}$ also used English, Mandarin, and ISTS for ANL measurements. They reported comparability of ANLs across different languages (English, Mandarin, and the ISTS) yet they questioned applicability of ISTS to serve as an international ANL material.

In our present study, we sought to investigate whether the ANLs would differ across different types of speech material (intelligible Korean speech, two unintelligible speech signals of reversed Korean speech and ISTS) under eight-talker babble noise in subjects with normal hearing. Here, the difference between two unintelligible signals is that the reversed speech signal retains similar spectral contents as Korean speech, and the ISTS consists of concatenated syllables obtained from six different languages. In the daily clinical setting, it can be impractical to perform ANL tests using various language speech signals. If our hypothesis that the ANLs would be independent of the meaningfulness of the target speech signal, it would support using a single speech material in the clinic for speakers of various languages.

\section{Subjects and Method}

\section{Participants}

Initially, 30 Korean participants were recruited for the study at Gangnam Severance Hospital, Seoul, Korea. Five participants did not meet the inclusion criteria and were excluded. Thus, 25 Korean participants ( 9 males and 16 females, mean age $28.5 \pm 3.15$ years) were included. The inclusion criteria were as follows: no known prior history of hearing loss or tinnitus or any neurologic disorders, normal otoscopic findings, hearing thresholds better than $15 \mathrm{~dB}$ HL from 250$8000 \mathrm{~Hz}$ at the octave frequencies and A-type tympanograms in both ears. The Institutional Review Board (IRB No. 3-2017-0124) of Hospital approved this study.

\section{Stimuli}

For ANL measurement, three different types of materials (Korean speech, reversed Korean speech, and ISTS) were used as target signal and a multi-talker (eight talkers) babble was used as background noise. The Korean speech and the multitalker babble were obtained from the previous study ${ }^{6}$ that developed the Korean-version ANL material initially. The Korean speech signal was recorded by male native Korean speaker, reading a story taken from an easy-to-read Korean history book. The book was written for the school-aged children and the stories were readily understandable to the adult listeners and their recordings could represent a typical daily listening environment. A recording of each story was conducted in a double-wall sound booth with a Computerized Speech Lab (CSL ${ }^{\mathrm{TM}}$, model 4500; Kay Elemetrics Corporation, Pentax, NJ, USA) speech analyzer and SM48-LC microphone (Shure, Niles, IL, USA). The reversed Korean signal was generated by playing the recorded Korean speech 


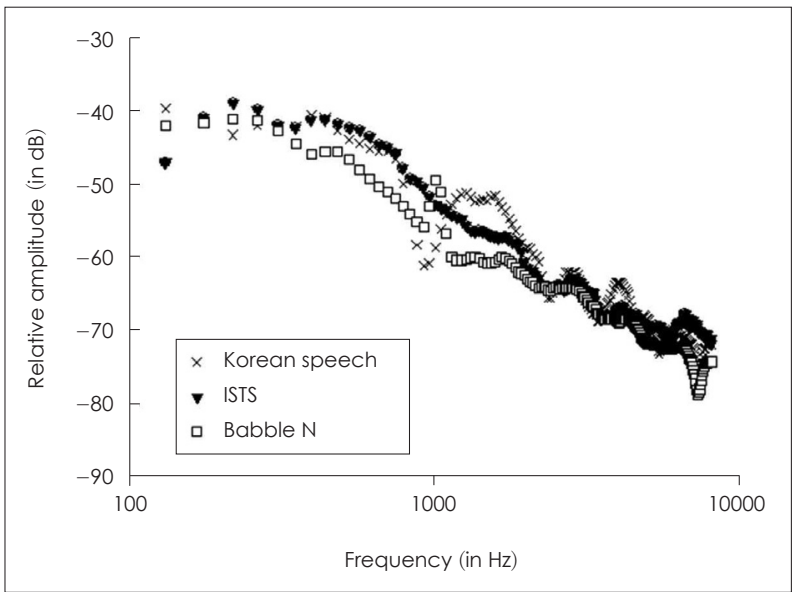

Fig. 1. Long-term average speech spectrum of Korean speech signal, ISTS, and eight-talker babble noise on a logarithmic scale. ISTS: international speech test signal.

signal $^{6)}$ reversed in time using Adobe Audition (ver 3.0; Adobe Systems Inc., San Jose, CA, USA). In this way, the reversed speech could have the same spectral content as in Korean speech, but as a non-meaningful (unintelligible) signal. Finally, the ISTS ${ }^{11)}$ was used as a target signal. The ISTS is composed of mixed short fragments of recordings of different languages. Thus, the ISTS sounds like speech but has no semantic content.

All the wavefiles were stored using a sampling rate of 44100 $\mathrm{Hz}$, mono channel and 16-bit resolution. To verify the equivalence of overall intensity across different stimuli, the average root-mean-squared values of all the signals were checked and equalized using Adobe Audition (ver. 3.0). The long-term average speech spectra of recorded signals were analyzed using 1024-point Fast Fourier Transform and BlackmannHarris window, as shown in Fig. 1.

\section{Equipment and procedure}

All tests were performed in a double-walled soundproof booth in accordance with American National Standards Institute S3.1-1999 (R 2008). Pure tone audiometry and tympanometry were conducted using Madsen ORBITER 922 diagnostic audiometer (GN Otometrics, Taastrup, Denmark) and Grason-Stadler Tympstar System (GSI Viasys Healthcare, Madison, WI, USA), respectively. For ANL measurements, all the signals were presented through loudspeaker (Aviano 1, Mordaunt-Short, London, UK) at $0^{\circ}$ azimuth 1 meter from the participant. The actual output levels of both target and noise signals delivered through transducer were verified using a sound level meter (Type 2150L, Brüel and Kjær, Skodsborgvej, Denmark). The A-weighted equivalent continuous noise level, denoted as LAeq, was measured over a specified time interval, at the position of the listener's head, such that all the signals were adjusted to have the same overall level, $65 \mathrm{~dB}$ sound pressure level.

The participants were given written and oral instructions before the ANL measurement. ANL testing was administered according to the procedure described in the study by $\mathrm{Na}$ belek, et al. ${ }^{1)}$ as follows. Given that the ANL could depend on the content of the instructions and the understanding of these instructions, three professional audiologists verified the content of ANL instruction in Korean. In addition, the tester checked whether each subject understood the instructions before beginning the measurements. Further instructions were given during the test session as needed. The initial presentation level was $30 \mathrm{~dB} H \mathrm{HL}$, and increased in $2 \mathrm{~dB}$ steps until the participant signaled that it was too loud. Then it was decreased in $5 \mathrm{~dB}$ steps until the signal was too soft. Then the step size was reduced from $5 \mathrm{~dB}$ step to $2 \mathrm{~dB}$ step for the final adjustment until MCL was identified. ${ }^{12)} \mathrm{MCL}$ measurements were repeated three times, and only the second and third measurements were averaged. Next, we measured the maximum BNL that listeners could accept while following the target speech signal at the average MCL. The target signal was fixed at the MCL level measured previously. Again, the participants signaled by thumb-up or thumbdown hand gesture to adjust the noise level in $5 \mathrm{~dB}$ steps, initially increasing until it became too loud, then decreasing until the speech became very clear. Again, $2 \mathrm{~dB}$ steps were used in final adjustment. ANL is then calculated as the difference between MCL and BNL (ANL=MCL-BNL). The two ANLs were averaged to serve as the ANL for the condition. The procedures were completed for each of the ANL stimuli (Korean speech, reversed Korean, and ISTS). The test order of the three stimuli was randomized.

\section{Data analysis}

Statistical analysis of the collected data was performed using SPSS version 15 (SPSS Inc., Chicago, IL, USA). To examine the effects of different types of speech signals on ANLs, analysis of variance (ANOVA) with repeated measures was conducted (alpha levels $<0.05$ were considered statistically significant). We used Bland-Altman plots to assess the degree of agreement between any pair of the three methods with respect to the measurements of the different ANL examined. Lin's concordance-correlation coefficient was used to evaluate the correlation between pairs of conditions (Korean vs. reversed 
Korean, Korean vs. ISTS, and reversed Korean and ISTS). ${ }^{13)}$

\section{Results}

Table 1 shows the mean MCL, BNL, and ANLs measured with three speech signals in the normal-hearing listeners. Generally, the mean MCL was approximately $45 \mathrm{~dB}$ HL and the mean BNLs was approximately $40 \mathrm{~dB}$ HL across three different speech materials. Also, the mean ANL did not differ significantly whether the Korean speech, the reversed speech, or the ISTS was given as the target signal. The results of one-way ANOVA with repeated measures revealed no significant difference in the MCL, BNL, and ANLs across three speech materials $(p>0.05)$, indicating that the meaningfulness of content in the target speech did not affect the individuals' MCL or noise tolerance level, at least for the normal-hearing young adults. Measurements of MCL, BNL, and ANLs were confirmed to satisfy both normality assumption and sphericity assumption. In addition, analysis of Pearson correlation was carried out to examine the relationships

Table 1. The mean MCL, BNL and ANL obtained using three different speech signals in normal hearing listeners $(n=25)$

\begin{tabular}{lrrr}
\hline \multicolumn{1}{c}{ Speech signals used } & Mean & SD & Range \\
\hline Korean speech & & & \\
MCL (dB HL) & 45.4 & 6.7 & $32-62$ \\
BNL (dB HL) & 39.6 & 8.0 & $27-61$ \\
ANL (dB) & 5.8 & 2.3 & $1-11$ \\
Reversed Korean speech & & & \\
MCL (dB HL) & 45.1 & 6.1 & $32-58$ \\
BNL (dB HL) & 39.4 & 8.4 & $26-55$ \\
ANL (dB) & 5.7 & 3.4 & $1-12$ \\
ISTS & & & \\
MCL (dB HL) & 45.1 & 6.6 & $30-58$ \\
BNL (dB HL) & 40.0 & 8.0 & $23-55$ \\
ANL (dB) & 5.1 & 2.8 & $2-13$ \\
\hline
\end{tabular}

MCL: maximum comfortable level, BNL: background noise level, ANL: acceptable noise level, ISTS: international speech test signal, SD: standard deviation
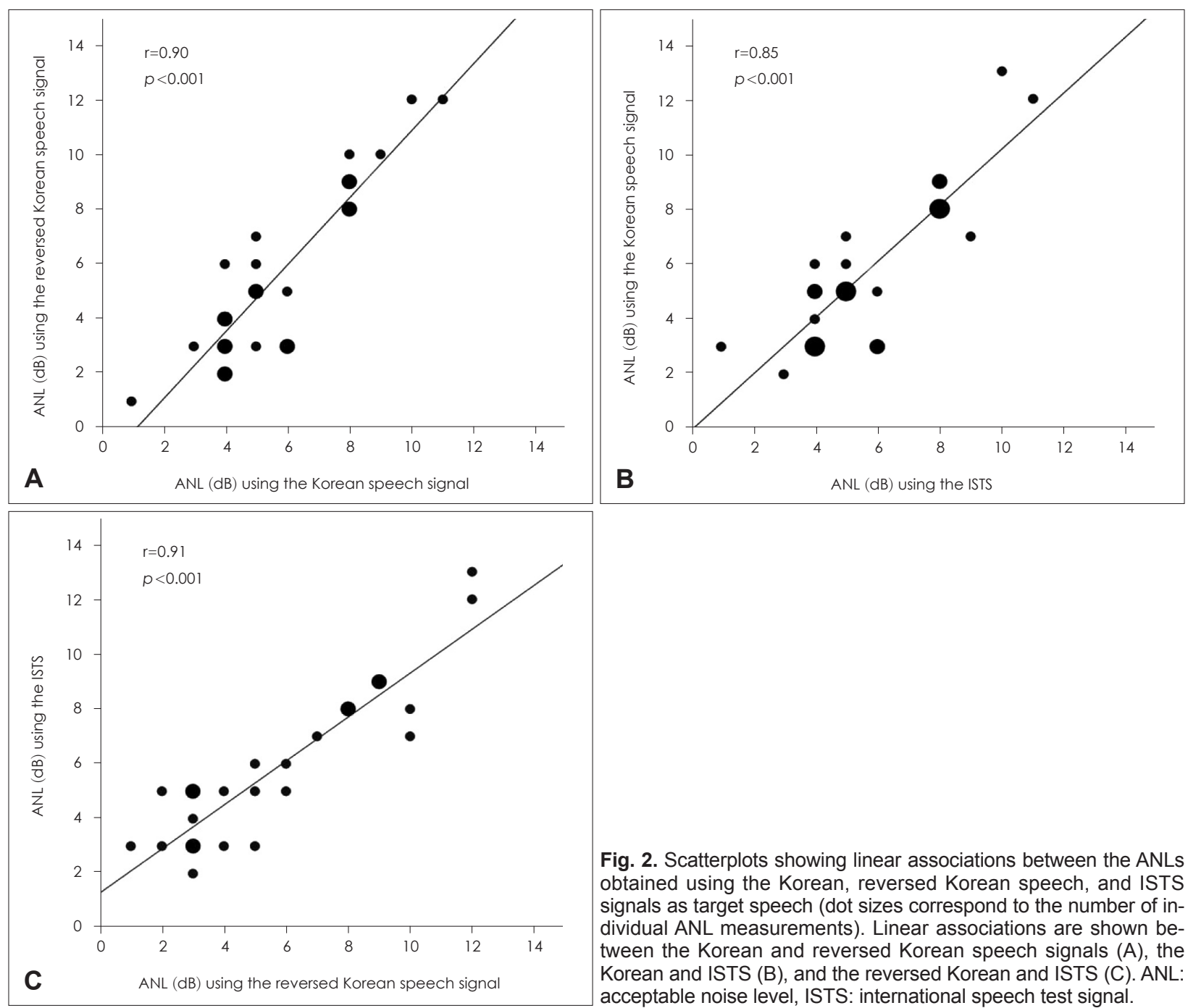

Fig. 2. Scatterplots showing linear associations between the ANLS obtained using the Korean, reversed Korean speech, and ISTS signals as target speech (dot sizes correspond to the number of individual ANL measurements). Linear associations are shown between the Korean and reversed Korean speech signals $(A)$, the Korean and ISTS (B), and the reversed Korean and ISTS (C). ANL: acceptable noise level, ISTS: international speech test signal. 

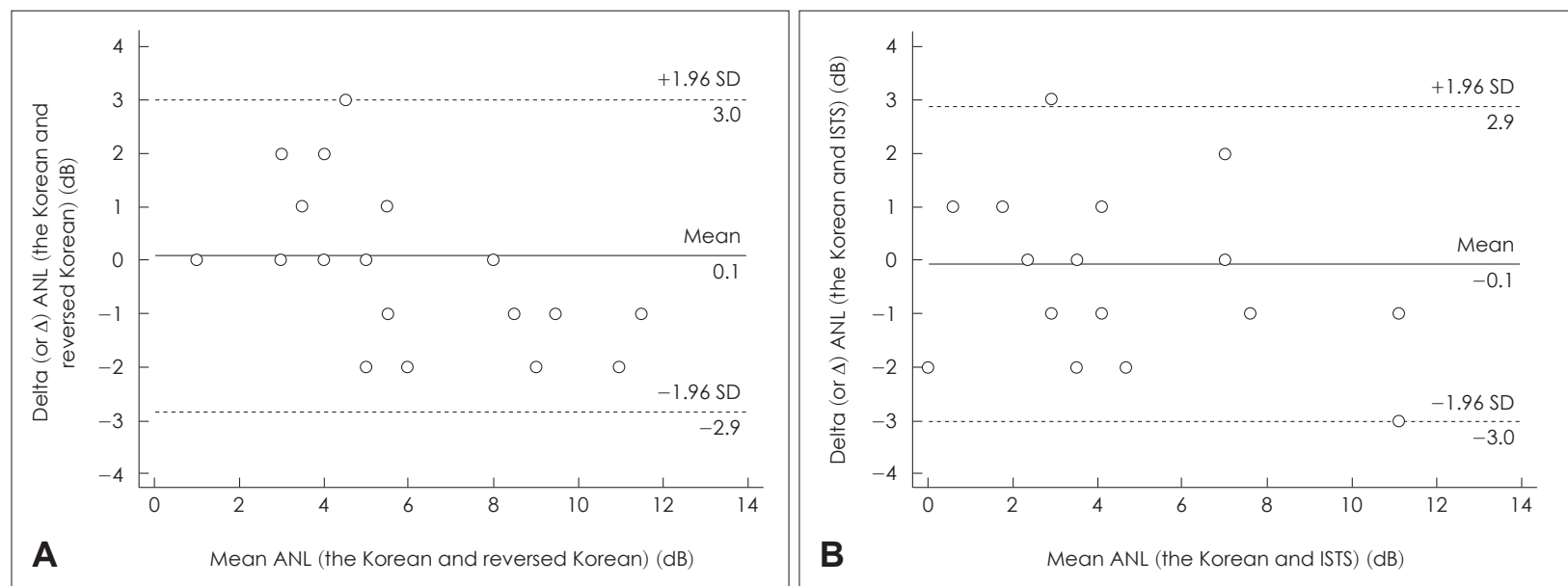

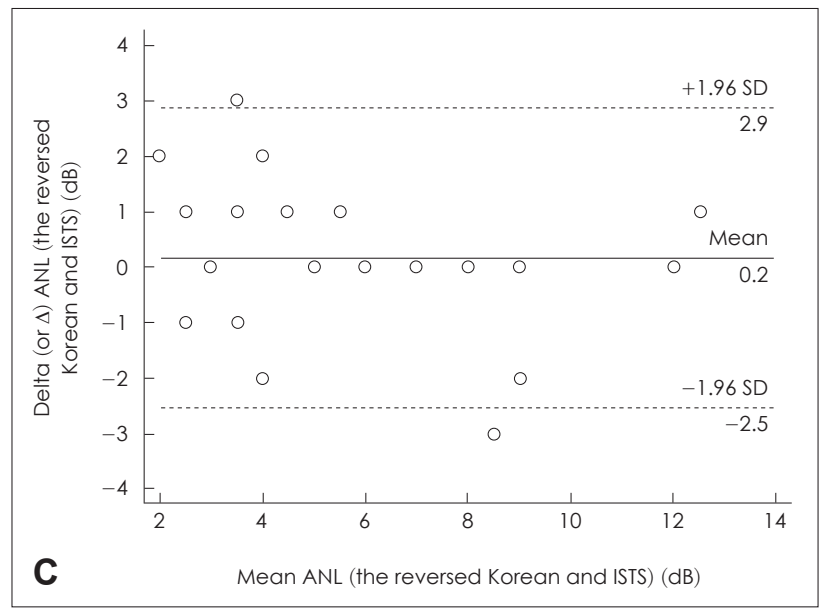

among ANLs obtained with Korean, reversed Korean, and ISTS signals. As shown in panel A of Fig. 2, the ANLs for Korean speech were significantly and strongly correlated with the ANLs for reversed Korean speech (Pearson correlation coefficient $\mathrm{r}=0.90, p<0.001)$. Panel B of Fig. 2 shows that the individuals with higher ANLs for the presentation of Korean speech signals also had higher ANLs when following the ISTS material (Pearson correlation coefficient $\mathrm{r}=0.85, p<0.001$ ). This tendency was similar when the ANLs for reversed Korean speech and ISTS signals were analyzed, as shown in panel C of Fig. 2 (Pearson correlation coefficient $\mathrm{r}=0.91, p$ $<0.001)$. This suggests that normal-hearing adults who can tolerate background noise more (lower ANL) when following one type of target signals can actually accept more background noise for other target signals, regardless of intelligibility or semantic content of target signal. Bland-Altman plots were used to compare ANLs measured using the three different speech signals (Fig. 3). The plots show that the ANLs are in a good agreement with respect to the mean values, albeit with some scattering within the limits of agree-
Fig. 3. Bland-Altman plots for acceptable noise level showing good agreement with respect to the mean values, while there is a notable scattering, as indicated by the broad bandwidth of the limits of agreement. Results for comparison between the three test conditions are shown: (A) the Korean and reversed Korean speech signals, (B) the Korean and ISTS speech signals, and (C) the reversed Korean and ISTS speech signals. ANL: acceptable noise level, ISTS: international speech test signal, SD: standard deviation.

Table 2. Lin's concordance-correlation coefficients comparing acceptable noise levels using Korean, reversed Korean speech signals, and ISTS

\begin{tabular}{lc}
\hline & $\begin{array}{c}\text { Lin's concordance } \\
\text { correlation coefficients }\end{array}$ \\
\hline Korean vs. reversed Korean & 0.8605 \\
Korean vs. ISTS & 0.8377 \\
Reversed Korean vs. ISTS & 0.8974 \\
\hline
\end{tabular}

ISTS: international speech test signal

ment. Lin's concordance-correlation coefficients are shown in Table 2. Again, the ANL measurements were comparable among the three speech signals.

\section{Discussion}

It is obvious that the listener's ability to identify speech in noise is affected by language intelligibility. However, since the ANL is a measurement of intrinsic characteristics that reflect the listener's acceptance of background noise, the listener is not required to understand the target story as long as the listener is able to "follow" the speech signal. Several stud- 
ies aimed to assess language effects on ANLs reported conflicting results. In one study, ANLs showed no difference between English and Korean speech in bilingual adults regardless of language proficiency. ${ }^{14)}$ However, the study of Gordon-Hickey \& Moore showed different results. ${ }^{15)}$ In their study, the MCL was increased in unfamiliar speech conditions (reversed English speech and Chinese speech), although the change was not statistically significant. However, the BNL was decreased in the reversed speech condition compared with intelligible speech condition, and unchanged in unfamiliar speech condition (Chinese language). The combined effect of these changes resulted in a small but significant increase in ANLs for both reversed and unfamiliar speech signals, suggesting that ANL may be affected by the intelligibility of the speech signal. When three different sets of Mandarin speech materials from primary, secondary and high school textbooks were used, ANL measurements did not show difference in normal hearing subjects. ${ }^{16)}$ Also, when comparing different English speech materials, it was reported that neither MCL nor ANL was different, suggesting that the content of the speech materials did not affect ANL in normal hearing subjects. ${ }^{17)}$ Another study used English, Mandarin, and ISTS for ANL measurements. ${ }^{7)}$ These authors reported that ANLs were comparable using native and foreign language in two distinct locations. In our study, ANLs did not differ among intelligible (Korean language) and non-intelligible (reversed Korean and ISTS) speech signals. It is possible that the listener's perceptions differed among different signals, such as a totally unknown or less familiar foreign language or reversed speech signal. Also, the discrepancies in these studies may be attributed to other factors potentially influencing ANLs, such as the noise selection, specific instructions, or cultural differences. ${ }^{8)}$

In our study, we addressed whether ISTS could be used as a universal test material. The ISTS consists of mixed short fragments across various recordings of different languages. Although it sounds like speech, it is not intelligible. Our study showed similar ANL measurements using ISTS and Korean language speech material. In a previous study, ANL measurements using Danish speech material and ISTS were comparable for Swedish listeners, while significant difference was shown between Danish speech material and ISTS in Danish listeners. ${ }^{9)}$ Another study showed that ANL measurements were similar between speech material in listener's primary language (English or Mandarin) and ISTS.') However, the ANL measurements using ISTS in two distinct centers in the US and Taiwan were not equivalent. ${ }^{7)}$ One issue raised by the authors was that the listeners may perceive ISTS as unnatural, thus they may be less willing to accept additional noise while listening to ISTS. Also their results suggested that the instructions given at each test locations may be another confounding factor. It was more difficult to explain the test procedure to the subject when using unintelligible signal as the speech signal. In our study, some of the subjects mentioned not being able to understand the non-semantic signals (either reversed Korean or ISTS), and the tester explained the instructions again. It is possible that an unfamiliar accent, a foreign language, manipulated speech (such as reversed or reverberation modulated speech), and the ISTS might be perceived differently by a listener. To accurately assess whether the intelligibility of speech material affects ANLs, the characteristics of the "unintelligible" speech signals should be taken into consideration. Also, different acoustic characteristic and speaking rate from primary language speech signals may contribute to discrepancies between primary language and ISTS ANLs. While it would be practical to use ISTS as the speech material across different language users, comparison with various languages is still needed before a generalization could be made.

Because ANL is a psychoacoustic measurement, it is important that the instructions (both written and verbal) are consistent in all clinics. ${ }^{18)}$ As in any audiological evaluation tool, translation of ANL instructions into the primary language in a particular population is necessary, and care must be taken when translating the test instructions into different languages. Translation or modifications of the instructions may affect the ANL results as shown previously. ${ }^{7)}$ By using a consistent set of written and step-by-step verbal instructions, the test subject and audiologist are guided to accurate measurement. Because ANL is a calculated score from two independent variables, measurement of these variables must be consistent. When consistent instructions are followed, ANLs have been shown to be a reliable measurement. ${ }^{2,3)}$ When unfamiliar speech signals are used instead of primary language speech signals, consistent instructions are again important to ensure that the subject understands the intent of the measurements, as the "speech" signal might be confused as a variation of "background noise."

When the ANL and other individual measurements are compared to normal hearing listener using the original English ANL test materials, the ANLs of the Korean listeners tended to be somewhat lower. ${ }^{4,1920)}$ Since the ANLs show high variability among normal hearing listenres and can be influenced by various factors such as the language of speech sig- 
nal, the type of the background noise used, presentation mode and response instructions, it is difficult to directly compare raw data across studies. Nevertheless, our measurements were comparable to previous studies using the same Korean version of ANL and following same instructions. ${ }^{6,20)}$ Further studies are needed to investigate the effect of language on ANL measurements.

In summary, ANLs were similar across Korean, reverse Korean, and ISTS signals as target when the normal hearing subjects followed the target speech signal against eight-talker babble noise. This result suggests the possibility of using non-semantic signals for ANL measurements.

\section{Acknowledgments}

We thank Dr. Hanna Yoo (Biostatistics Collaboration Unit, Yonsei University College of Medicine) for assistance in the statistical analyses.

Work described in this manuscript is supported by the National Research Foundation of Korea (Grant 2013R1A1A2007622 to E.J.S. and a faculty research grant of Yonsei University College of Medicine (6-2012-0023 to E.J.S.).

\section{REFERENCES}

1) Nabelek AK, Tucker FM, Letowski TR. Toleration of background noises: relationship with patterns of hearing aid use by elderly persons. J Speech Hear Res 1991;34(3):679-85.

2) Nabelek AK, Freyaldenhoven MC, Tampas JW, Burchfiel SB, Muenchen RA. Acceptable noise level as a predictor of hearing aid use. J Am Acad Audiol 2006;17(9):626-39.

3) Nabelek AK, Tampas JW, Burchfield SB. Comparison of speech perception in background noise with acceptance of background noise in aided and unaided conditions. J Speech Lang Hear Res 2004;47(5): 1001-11.

4) Rogers DS, Harkrider AW, Burchfield SB, Nabelek AK. The influence of listener's gender on the acceptance of background noise. J Am Acad Audiol 2003;14(7):372-82; quiz 401.

5) Walravens E, Keidser G, Hartley D, Hickson L. An Australian version of the acceptable noise level test and its predictive value for successful hearing aid use in an older population. Int J Audiol 2014;53 Suppl 1: S52-9.

6) Shin JB, Lee JH. Effects of the target talker gender and the number of competing talkers on acceptable noise level (ANL) of Korean normal-hearing adults. Audiology and Speech Research 2010;6(2): 146-52.

7) Ho HC, Wu YH, Hsiao SH, Stangl E, Lentz EJ, Bentler RA. The equivalence of acceptable noise level (ANL) with English, Mandarin, and non-semantic speech: a study across the U.S. and Taiwan. Int J Audiol 2013;52(2):83-91.

8) Brännström KJ, Lantz J, Nielsen LH, Olsen SØ. Acceptable noise level with Danish, Swedish, and non-semantic speech materials. Int J Audiol 2012;51(3):146-56.

9) Olsen SØ, Nielsen LH, Lantz J, Brännström KJ. Acceptable noise level: repeatability with Danish and non-semantic speech materials for adults with normal hearing. Int J Audiol 2012;51(7):557-63.

10) Olsen SØ, Lantz J, Nielsen LH, Brännström KJ. Acceptable noise level (ANL) with Danish and non-semantic speech materials in adult hearing-aid users. Int J Audiol 2012;51(9):678-88.

11) Holube I, Fredelake S, Vlaming M, Kollmeier B. Development and analysis of an international speech test signal (ISTS). Int J Audiol 2010;49(12):891-903.

12) Gordon-Hickey S, Adams E, Moore R, Gaal A, Berry K, Brock S. Intertester reliability of the acceptable noise level. J Am Acad Audiol 2012;23(7):534-41.

13) Lin LI. A concordance correlation coefficient to evaluate reproducibility. Biometrics 1989;45(1):255-68.

14) von Hapsburg D, Bahng J. Acceptance of background noise levels in bilingual (Korean-English) listeners. J Am Acad Audiol 2006;17 (9):649-58.

15) Gordon-Hickey S, Moore RE. Acceptance of noise with intelligible, reversed, and unfamiliar primary discourse. Am J Audiol 2008;17 (2):129-35.

16) Chen J, Zhang H, Plyler PN, Cao W, Chen J. Development and evaluation of the Mandarin speech signal content on the acceptable noise level test in listeners with normal hearing in mainland China. Int J Audiol 2011;50(6):354-60.

17) Plyler PN, Alworth LN, Rossini TP, Mapes KE. Effects of speech signal content and speaker gender on acceptance of noise in listeners with normal hearing. Int J Audiol 2011;50(4):243-8.

18) Olsen SØ, Brännström KJ. Does the acceptable noise level (ANL) predict hearing-aid use? Int J Audiol 2014;53(1):2-20.

19) Franklin CA Jr, Thelin JW, Nabelek AK, Burchfield SB. The effect of speech presentation level on acceptance of background noise in listeners with normal hearing. J Am Acad Audiol 2006;17(2):141-6.

20) Kim JH, Lee JH, Lee HK. Advantages of binaural amplification to acceptable noise level of directional hearing aid users. Clin Exp Otorhinolaryngol 2014;7(2):94-101. 